\title{
Lactic acid concentrations in bronchoalveolar lavage fluid correlate with neutrophil influx in cystic fibrosis
}

\author{
Keith C Meyer*, Amy Amessoudji, Trina Hollatz and Francis HS Tsao \\ Department of Medicine, University of Wisconsin School of United States, USA
}

\begin{abstract}
Because oxygen tension can be extremely low in respiratory secretions of patients with cystic fibrosis (CF), host inflammatory cells in the airspace milieu may resort to anaerobic metabolism with production of lactic acid under hypoxic conditions. We found elevated lactate levels in bronchoalveolar lavage (BAL) fluid specimens obtained from patients with $\mathrm{CF}$, and lactate concentrations correlated with neutrophil and neutrophil-derived mediator concentrations. We suggest that neutrophils, particularly when present in high numbers, may resort to anaerobic metabolism due to hypoxia in CF respiratory secretions and generate lactate, which may be a useful biomarker of inflammation in the CF lung.
\end{abstract}

\section{Introduction}

Cystic fibrosis transmembrane conductance regulator (CFTR) dysfunction causes impaired transepithelial ion flux, depletion of airway surface liquid, and impaired mucociliary clearance in the cystic fibrosis (CF) lung [1,2]. These consequences of CFTR dysfunction allow chronic infection with opportunistic bacteria (such as Pseudomonas aeruginosa and Staphylococcus aureus) and sustained inflammation to become established in the lungs of patients with cystic fibrosis (CF) early in life [3-5], leading to gradual remodeling and destruction of the CF lung, progressive decline in lung function, and diminished longevity [2].

Airspace inflammation in the adult CF lung is characterized by the influx of massive numbers of neutrophils [5-8], and neutrophildominated inflammation has been implicated as a key factor in airway remodeling that leads to bronchiectasis [2,9] as well as elastin loss and collagen deposition in alveoli [10]. Additionally, accumulated mucus in CF airways is characterized by extreme hypoxia, which can provide an anaerobic environment that promotes persistence of $P$. aeruginosa in CF airways and alginate production [11]. Esther, et al. [12] have identified numerous metabolites in CF bronchoalveolar lavage (BAL) fluid that are related to neutrophilic inflammation, and lactate concentrations have been shown to be elevated in sputa [13] and BAL fluid [14] from CF patients, and neutrophils appear to be the predominant source of lactate [13].

We hypothesized that lactate concentrations may be increased in airspaces of the CF lung and that concentrations of lactate in BAL fluid may correlate with the intensity of the neutrophil-dominated inflammation that is associated with chronic infection and an anaerobic microenvironment. Therefore, we measured lactate levels in BAL fluid from adult patients with $\mathrm{CF}$ and correlated lactate concentrations with levels of neutrophils, neutrophil-derived mediators, and $P$. aeruginosa density.

\section{Methods}

\section{Patients}

This research was approved by the University of Wisconsin Human Subjects Committee, and written informed consent was obtained from all volunteers. Seventeen patients with cystic fibrosis had bronchoscopy and BAL. Four females and 13 males were included with a mean age of $23 \pm 7$ [mean \pm SD] (range 17-44). The mean baseline FEV1 percent predicted was $62.1 \% \pm 20.3 \%$ SD (range $34-100 \%$ ). Seven of the patients with chronic persistence of $P$. aeruginosa in their respiratory secretions had BAL performed just before and at the end of a 2-week course of intravenous anti-pseudomonal antibiotics for respiratory exacerbations as previously described [6]. The remaining 10 subjects were clinically stable outpatients and underwent bronchoscopy with BAL performed in the upper and lower lobe regions as previously described [15]. All the subjects with CF had more severe disease on routine postero-anterior and lateral chest radiographs in upper lung versus lower lung zones. BAL fluid specimens obtained from 15 young adults (age range 18-35 years) without CF were also analyzed; these healthy volunteers had never smoked, had normal BAL differential cell counts, and had normal spirometry values.

\section{Specimen handling and analyses}

Sequential aliquots of BAL fluid obtained from specific target areas were filtered through loose gauze to remove mucus plugs, pooled, and then centrifuged at $400 \mathrm{x} g$ for $10 \mathrm{~min}$ at $22^{\circ} \mathrm{C}$. Aliquots of BAL supernatant fluid were then frozen at $-70^{\circ} \mathrm{C}$ until assayed. BAL samples were analyzed for neutrophil concentration, neutrophil elastase (NE), myeloperoxidase (MPO), lactate levels and $\mathrm{CFU} / \mathrm{ml}$ of $P$. aeruginosa as previously described [6]. Briefly, neutrophil concentrations were enumerated via hemocytometer and cytocentrifuge slide

${ }^{\star}$ Correspondence to: Keith C Meyer, MD, MS, FCCP, Professor of Medicine, University of Wisconsin Lung Transplant and Advanced Lung Disease Program Section of Allergy, Pulmonary and Critical Care Medicine, Department of Medicine, University of Wisconsin School of Medicine and Public Health, USA, Tel: 608 263-6363; 608 263-3035; FAX: 608 263-3104; E-mail: kcm@medicine.wisc.edu

Key words: lactate, cystic fibrosis, bronchoalveolar lavage, neutrophils, metabolomics, biomarker

Received: November 05, 2018; Accepted: November 20, 2018; Published: November 27, 2018 
preparations of cell suspensions. NE activity was determined via spectrophotometry using MeO-Suc-Ala-Ala-Pro-Val-pNA as a specific substrate. MPO activity was quantified spectrophotometrically using 3,3',5,5'-tetramethyl benzidine (TMB) as the substrate. Dilutions of BAL fluid was plated on appropriate media to identify and obtain quantitative colony counts of aerobic bacteria.

\section{Lactate measurement}

Lactate content in BAL fluid was determined using a Lactate Reagent Kit (Trinity Biotech USA, St. Louis, MO) that was modified for microplate assays. An aliquot of $10 \mu$ of diluted BAL fluid solution ( 21 of BAL fluid and $8 \mu \mathrm{l}$ deionized water) was added to the wells of a 96-well microplate. An aliquot of $0.2 \mathrm{ml}$ of lactate reagent solution was then transferred to the well that contained BAL fluid samples and the solution was mixed well. Each BAL fluid sample was assayed in duplicate. Graded concentrations of lactate standard solution (20, $40,60,80$, and $100 \mathrm{mg} / \mathrm{dl}$ ) were assayed in parallel and in duplicate. The plate was set at room temperature for 5-10 min, and the optical density of the solution was determined at $540 \mathrm{~nm}$ using a plate reader. Lactate concentration in the BAL fluid was calculated from the BAL fluid optical density and standard curve and expressed as $\mathrm{mg} / \mathrm{dl}$ of BAL fluid.

\section{Statistical analysis}

Data are expressed as mean \pm standard of the mean as appropriate. Values were analyzed via independent or paired t-tests, and correlations were made via Spearman Rank Order testing.

\section{Results}

Lactate concentrations were detectable in 17 of $34 \mathrm{BAL}$ fluid specimens, and BAL lactate concentrations correlated significantly with neutrophil number $(\mathrm{R}=0.624, \mathrm{p}<0.001$ by Spearman Rank Order testing) (Figure 1) and neutrophil-derived mediators (MPO \& NE) concentrations in BAL fluid (data not shown). In contrast, BAL lactate concentrations did not correlate as well with $\mathrm{CFU} / \mathrm{ml}$ of $P$. aeruginosa $(\mathrm{R}=0.405, \mathrm{p}=0.02)$ (Figure 2). Lactate was not detected in any BAL fluid specimens from normal subjects.

Regional BAL lactate concentrations in clinically stable outpatients who had BAL performed in both an upper and lower lobe region were $80.2 \pm 27.5 \mu \mathrm{g} / \mathrm{ml}$ for upper lobe BAL vs. $11.6 \pm 9.9 \mu \mathrm{g} / \mathrm{ml}$ for lower lobe BAL ( $\mathrm{p}=0.008)$. BAL neutrophil concentrations were $69.6 \pm 13.9$ x $10^{5} / \mathrm{ml}$ for upper lobe lavages versus $0.96 \pm 0.7 \times 10^{5} / \mathrm{ml}$ for lower lobe lavage ( $\mathrm{p}<0.001$ ) (Figure 3 ). Lactate concentrations in upper lobe specimens correlated significantly with upper lobe BAL neutrophil concentrations $(\mathrm{R}=0.729, \mathrm{p}<0.001$, Spearman Rank Order), but lower lobe BAL lactate versus neutrophil concentrations were not significantly correlated $(\mathrm{R}=0.307, \mathrm{p}=0.31)$.

For the 7 patients who underwent BAL when admitted to the hospital for treatment of a respiratory exacerbation and had a repeat BAL performed after 2 weeks of broad-spectrum, anti-pseudomonal intravenous antibiotic therapy, four subjects had significantly elevated lactate concentrations in the first BAL. BAL lactate concentrations declined significantly $(55.1 \pm 33.0$ to $1.0 \pm 1.0 \mu \mathrm{g} / \mathrm{ml}$ for these 4 patients. However, one of the 3 patients without detectable lactate in the initial BAL specimen had a high concentration of lactate in the second BAL specimen that was accompanied by a considerable increase in BAL neutrophils and a lack of response (lung function \& symptoms) to the 2-week course of intravenous antibiotics (Supplementary Figures 1-4).

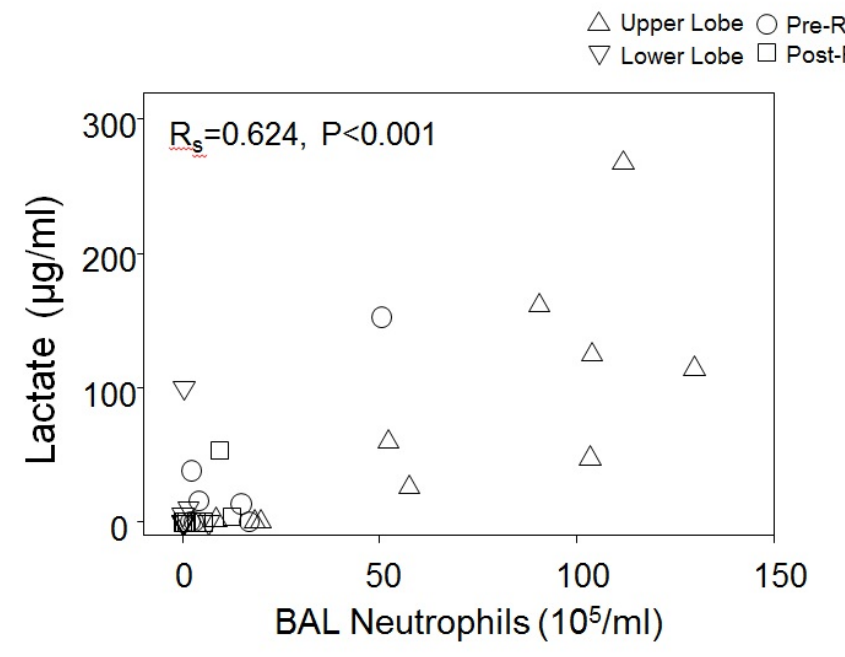

Figure 1. Lactate versus neutrophil concentrations for all BAL specimens

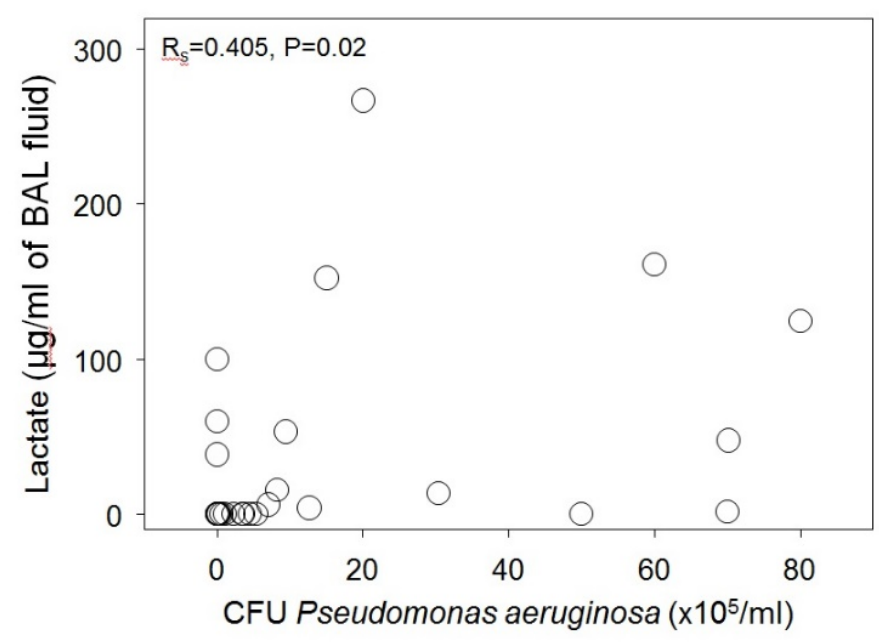

Figure 2. Lactate concentrations versus colony forming units (CFU) of $P$. aeruginosa in all BAL specimens
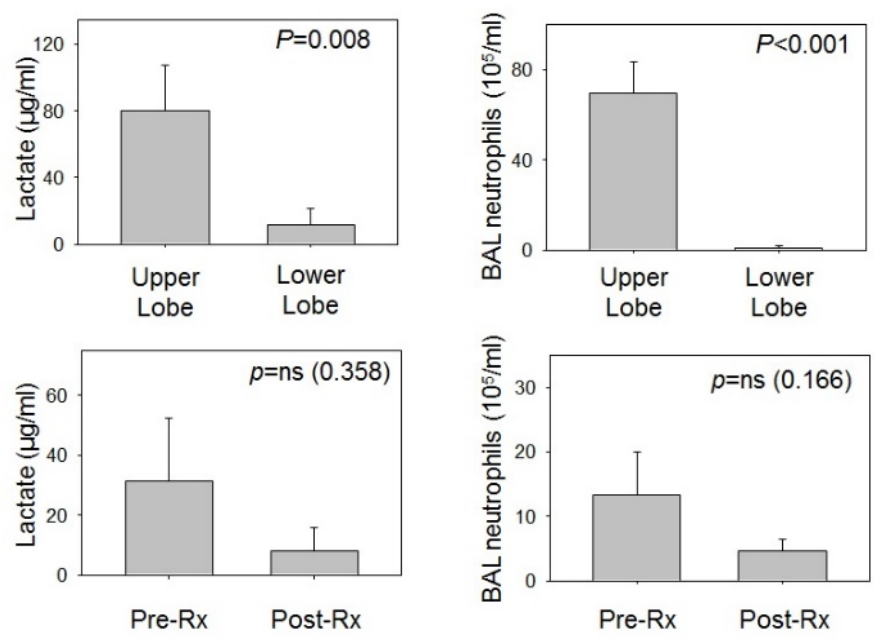

Figure 3. Lactate and neutrophil concentrations for upper lobe versus lower lobe regions (stable outpatients) and for before and after intravenous broad-spectrum antibiotics (patients admitted to hospital for respiratory exacerbation requiring antibiotic therapy) 


\section{Discussion}

Our study demonstrates that high concentrations of lactate can be detected in BAL fluid from adult patients with CF and that lactate concentrations positively correlate with the degree of BAL neutrophil content and concentrations of neutrophil mediators. Additionally, BAL lactate and neutrophil concentrations were much higher in upper lung regions, which tend to have more severe bronchiectatic changes [16] and have substantially more intense neutrophilic inflammation [15], versus lower lung regions. Finally, increased lactate concentrations were found in 4 of 7 patients with respiratory exacerbations just prior to receiving intravenous antibiotic therapy, and both lactate levels and neutrophil concentrations declined in BAL obtained from the same, initially lavaged lung segment at the end of a 2-week period of intravenous anti-pseudomonal antibiotic therapy. Interestingly, an additional patient did not respond clinically to antibiotics, and the second BAL showed an increase in neutrophils that was associated with the detection of lactate (which was not detectable in the first BAL specimen).

Airspace secretions from patients with CF have been shown to be profoundly hypoxic [11] such that prokaryotic (bacteria) or eukaryotic (neutrophils) cells in this milieu must be able to tolerate anaerobic conditions to survive and persist in this environment. Large numbers of neutrophils constantly flux into the airspaces of the CF lung and eventually undergo apoptosis and necrosis [17,18]. We suggest that these neutrophils have dysregulated cellular metabolism and must resort to glycolysis and generate lactate under such anaerobic conditions. This may then lead to the accumulation of lactic acid in the cytoplasm of neutrophils, which is released when neutrophils become necrotic, and lactate has been identified as a biomarker of anaerobic cellular metabolism [19]. However, lactate may also be derived from other tissue sources that are not directly linked to tissue hypoxia in the inflamed CF lung. Additionally, lack of oxygen in CF secretions may contribute to the impaired respiratory burst that has been described for neutrophils obtained from CF sputum [18] and CF BAL [20,21].

Lindy, et al. [22] reported that lactate concentrations were increased in lung tissues and preceded collagen deposition in a rat model of silica-induced fibrosis, and they attributed this increase to increased lactate dehydrogenase-5 (LDH-5) activity induced by tissue hypoxia. Additionally, lactate can be produced in the acutely injured lung [23]. Kottmann, et al. [24] recently observed that lactate levels are increased in lung tissue from patients with idiopathic pulmonary fibrosis (IPF), and these investigators demonstrated that lactic acid-induced myofibroblast differentiation via activation of transforming growth factor- $\beta$ was also associated with a subsequent increase in hypoxiainducible factor- $1 \alpha$ and LDH-5 expression. Lung tissue remodeling also occurs in the CF lung and is associated with increased numbers of myofibroblasts and considerably increased expression of nuclear factor- $\mathrm{\kappa} \mathrm{B}$, insulin-like growth factor-1, and intercellular adhesion molecule-1 [25]. We suggest that increased lactate in lung tissues and airspace secretions may promote or contribute to fibrogenesis with consequent destructive lung remodeling in both CF and IPF [26]. Additionally, we suggest that impaired perfusion of lung regions due to disrupted microcirculation that has been described for both IPF $[27,28]$ and the bronchiectatic lung $[29,30]$ may play a significant role in tissue hypoxia, cell turnover due to apoptosis and necrosis, and lactic acid generation.

In summary, we have shown that lactate can accumulate in airspace secretions in the CF lung and that lactate levels show a significant correlation with the degree of neutrophil influx as reflected by neutrophil numbers and neutrophil-derived mediator concentrations in BAL fluid. Increased lactate levels may reflect hypoxic conditions that promote persistence of a multitude of anaerobic flora that exist in the inflamed CF lung as well as microaerophilic organisms such as $P$. aeruginosa and Staphylococcus aureus [31]. Also, increased lactate concentrations are likely to promote acidification with lowering of the $\mathrm{pH}$ of airway secretions, and airway surface liquid acidification has been shown to impair host defense mechanisms and increase airway bacterial burden when CFTR is dysfunctional [32] Additionally, increased lactate concentrations may promote altered myofibroblast activity and pathologic remodeling of the CF lung, and inhibition of lactate production via inhibition of LDH by Gossypol has been shown to prevent TGF- $\beta$-mediated myofibroblast differentiation and collagen deposition [33]. We suggest that lactate levels may serve as a biomarker of neutrophilic influx and inflammation in CF lung disease, and that high concentrations of lactate may indicate increased risk of pathologic remodeling in the lungs of patients with $\mathrm{CF}$ and may also promote persistent bacterial infection.

\section{Financial/nonfinancial disclosures}

Dr. Meyer has been an investigator in clinical trials sponsored by Abbott, Actelion, Altana, Amgen, Asthmatx, Bayer, BoehringerIngelheim, Bristol Meyers Squibb, Chiron, Discovery Labs, DuPont Merck, Fibrogen, Genentech, Gilead, GlaxoSmithKline, Inspire, InterMune, Johnson \& Johnson, Novartis, Nycomed, Pfizer, Pharmaxis, PreAnalytiX, Roche, Ross, Vertex, and Wyeth. Dr. Meyer has also served on a Clinical Advisory Board for InterMune and serves on a clinical trial adjudication committee for Medimmune. All authors do not report any other relevant affiliations or financial involvement with any organization or entity with a financial interest in or financial conflict with the subject matter or materials discussed in the manuscript apart from those disclosed. No writing assistance was utilized in the production of this manuscript.

\section{Acknowledgment}

Supported in part by the George and Julie Mosher Pulmonary Research Fund.

\section{References}

1. Ratjen F, Döring G (2003) Cystic fibrosis. Lancet 361: 681-689 [Crossref].

2. Cantin AM, Hartl D, Konstan MW, Chmiel JF (2015) Inflammation in cystic fibrosis lung disease: Pathogenesis and therapy. J Cyst Fibros 14: 419-430 [Crossref].

3. Birrer P, McElvaney NG, Rüdeberg A, Sommer CW, Liechti-Gallati S, et al. (1994) Protease-antiprotease imbalance in the lungs of children with cystic fibrosis. $\mathrm{Am} \mathrm{J}$ Respir Crit Care Med 150: 207-213 [Crossref].

4. Armstrong DS, Grimwood K, Carlin JB, Carzino R, Gutièrrez JP, et al. (1997) Lower airway inflammation in infants and young children with cystic fibrosis. Am J Respir Crit Care Med 156: 1197-204.

5. Pillarisetti N, Williamson E, Linnane B, Skoric B, Robertson CF, et al. (2011) Australian Respiratory Early Surveillance Team for Cystic Fibrosis (AREST CF). Infection, inflammation, and lung function decline in infants with cystic fibrosis. Am J Respir Crit Care Med 184: 75-81.

6. Meyer KC, Lewandoski JR, Zimmerman JJ, Nunley D, Calhoun WJ, et al. (1991) Human neutrophil elastase and elastase/alpha 1-antiprotease complex in cystic fibrosis. Comparison with interstitial lung disease and evaluation of the effect of intravenously administered antibiotic therapy. Am Rev Respir Dis 144: 580-585 [Crossref].

7. Konstan MW, Hilliard KA, Norvell TM, Berger M (1994) Bronchoalveolar lavage findings in cystic fibrosis patients with stable, clinically mild lung disease suggest ongoing infection and inflammation. Am J Respir Crit Care Med 150: 448-454 [Crossref]. 
8. Meyer KC, Zimmerman J (1993) Neutrophil mediators, Pseudomonas, and pulmonary dysfunction in cystic fibrosis. J Lab Clin Med 121: 654-661 [Crossref].

9. Chmiel JF, Berger M, Konstan MW (2002) The role of inflammation in the pathophysiology of CF lung disease. Clin Rev Allergy Immunol 23: 5-27 [Crossref].

10. Ulrich M, Worlitzsch D, Viglio S, Siegmann N, Iadarola P, et al. (2010) Alveolar inflammation in cystic fibrosis. J Cyst Fibros 9: 217-227.

11. Worlitzsch D, Tarran R, Ulrich M, Schwab U, Cekici A, et al. (2002) Effects of reduced mucus oxygen concentration in airway Pseudomonas infections of cystic fibrosis patients. J Clin Invest 109: 317-325 [Crossref].

12. Esther CR Jr, Coakley RD, Henderson AG, Zhou YH, Wright FA, et al. (2015) Metabolomic Evaluation of Neutrophilic Airway Inflammation in Cystic Fibrosis. Chest 148:507-15.

13. Bensel T, Stotz M, Borneff-Lipp M, Wollschläger B, Wienke A, et al. (2011) Lactate in cystic fibrosis sputum. J Cyst Fibros 10: 37-44 [Crossref].

14. Wolak JE, Esther CR Jr, O'Connell TM (2009) Metabolomic analysis of bronchoalveolar lavage fluid from cystic fibrosis patients. Biomarkers 14: 55-60.

15. Meyer KC, Sharma A (1997) Regional variability of lung inflammation in cystic fibrosis. Am J Respir Crit Care Med 156: 1536-1540 [Crossref].

16. Li Z, Sanders DB, Rock MJ, Kosorok MR, Collins J, et al. (2012) Regional differences in the evolution of lung disease in children with cystic fibrosis. Pediatr Pulmonol 47: 635-640 [Crossref].

17. Tsao FH, Xiang Z, Abbasi A, Meyer KC (2012) Neutrophil necrosis and annexin 1 degradation associated with airway inflammation in lung transplant recipients with cystic fibrosis. BMC Pulm Med 12:44.

18. Houston N, Stewart N, Smith DS, Bell SC, Champion AC, et al. (2013) Sputum neutrophils in cystic fibrosis patients display a reduced respiratory burst. J Cyst Fibros 12: 352-362 [Crossref]

19. Loiacono LA, Shapiro DS (2010) Detection of hypoxia at the cellular level. Crit Care Clin 26: 409-421 [Crossref]

20. Meyer K, Zimmerman J, Lewandoski J (1991) Neutrophil-derived mediators and lower respiratory tract inflammation and dysfunction in CF. Pediatr Pulmonol 6: 300.

21. Meyer KC, Rosenthal N, Lewandoski J, Murphy K, Zimmerman J. Lower respiratory tract oxidant generation in cystic fibrosis. Chest 102: $81 \mathrm{~S}$.
22. Lindy S, Kahanpää K, Karhunen P, Halme J, Uitto J (1970) Lactate dehydrogenase isoenzymes during the development of experimental fibrosis. J Lab Clin Med 76: 756760 [Crossref].

23. De Backer D1, Creteur J, Zhang H, Norrenberg M, Vincent JL (1997) Lactate production by the lungs in acute lung injury. Am J Respir Crit Care Med 156: 10991104 [Crossref].

24. Kottmann RM, Kulkarni AA, Smolnycki KA, Lyda E, Dahanayake T, et al. (2012) Lactic acid is elevated in idiopathic pulmonary fibrosis and induces myofibroblast differentiation via $\mathrm{pH}$-dependent activation of transforming growth factor- $\mathrm{B}$. Am J Respir Crit Care Med 186: 740-751.

25. Ulrich M1, Worlitzsch D, Viglio S, Siegmann N, Iadarola P, et al. (2010) Alveolar inflammation in cystic fibrosis. J Cyst Fibros 9: 217-227 [Crossref].

26. Worlitzsch D, Meyer KC, Döring G (2013) Lactate levels in airways of patients with cystic fibrosis and idiopathic pulmonary fibrosis. Am J Respir Crit Care Med 188: 111 [Crossref].

27. Cosgrove GP, Brown KK, Schiemann WP, Serls AE, Parr JE, et al. (2004) Pigment epithelium-derived factor in idiopathic pulmonary fibrosis: a role in aberrant angiogenesis. Am J Respir Crit Care Med 170: 242-251.

28. Farkas L, Kolb M (2011) Pulmonary microcirculation in interstitial lung disease. Proc Am Thorac Soc 8: 516-521 [Crossref].

29. Liebow AA, Hales MR, Lindskog GE (1949) Enlargement of the bronchial arteries, and their anastomoses with the pulmonary arteries in bronchiectasis. Am J Pathol 25 211-231[Crossref].

30. Ashour M (1996) Hemodynamic alterations in bronchiectasis: a base for a new subclassification of the disease. J Thorac Cardiovasc Surg 112: 328-334 [Crossref].

31. Yonker LM, Cigana C, Hurley BP, Bragonzi A (2015) Host-pathogen interplay in the respiratory environment of cystic fibrosis. J Cyst Fibros 14: 431-439 [Crossref].

32. Shah VS, Meyerholz DK, Tang XX, Reznikov L, Abou Alaiwa M, et al. (2016) Airway acidification initiates host defense abnormalities in cystic fibrosis mice. Science 351 : 503-507.

33. Kottmann RM, Trawick E, Judge JL, Wahl LA, Epa A, et al. (2015) Pharmacologic inhibition of lactate production prevents myofibroblast differentiation. Am J Physiol Lung Cell Mol Physiol 309: L1305-12.

Copyright: $\odot 2018$ Meyer KC. This is an open-access article distributed under the terms of the Creative Commons Attribution License, which permits unrestricted use, distribution, and reproduction in any medium, provided the original author and source are credited. 